\title{
Quantum Degenerate Dipolar Fermi Gas
}

\author{
Mingwu Lu, ${ }^{1,2,3}$ Nathaniel Q. Burdick, ${ }^{1,2,3}$ and Benjamin L. Lev ${ }^{2,3,4}$ \\ ${ }^{1}$ Department of Physics, University of Illinois at Urbana-Champaign, Urbana, Illinois 61801, USA \\ ${ }^{2}$ Department of Applied Physics, Stanford University, Stanford, California 94305, USA \\ ${ }^{3}$ E. L. Ginzton Laboratory, Stanford University, Stanford, California 94305, USA \\ ${ }^{4}$ Department of Physics, Stanford University, Stanford, California 94305, USA
}

(Received 13 March 2012; published 21 May 2012)

\begin{abstract}
We report the first quantum degenerate dipolar Fermi gas, the realization of which opens a new frontier for exploring strongly correlated physics and, in particular, quantum liquid crystalline phases. A quantum degenerate Fermi gas of the most magnetic atom ${ }^{161}$ Dy is produced by laser cooling to $10 \mu \mathrm{K}$ before sympathetically cooling with ultracold, bosonic ${ }^{162} \mathrm{Dy}$. The temperature of the spin-polarized ${ }^{161} \mathrm{Dy}$ is a factor $T / T_{F}=0.2$ below the Fermi temperature $T_{F}=300 \mathrm{nK}$. The cotrapped ${ }^{162}$ Dy concomitantly cools to approximately $T_{c}$ for Bose-Einstein condensation, thus realizing a novel, nearly quantum degenerate dipolar Bose-Fermi gas mixture. Additionally, we achieve the forced evaporative cooling of spin-polarized ${ }^{161}$ Dy without ${ }^{162}$ Dy to $T / T_{F}=0.7$. That such a low temperature ratio is achieved may be a first signature of universal dipolar scattering.
\end{abstract}

DOI: 10.1103/PhysRevLett.108.215301

PACS numbers: 67.85.Hj, 03.75.Ss

The interplay between crystallinity and superfluidity is of great fundamental and technological interest in condensed matter settings. In particular, electronic quantum liquid crystallinity arises in the non-Fermi liquid, pseudogap regime neighboring a cuprate's unconventional superconducting phase as well as strontium ruthenates, 2D electron gases, and iron-based superconductors [1,2]. Such phases tend to arise under competition between shortand long-range interactions and are characterized as spontaneously breaking rotational and translational (or point group) symmetries of the Fermi surface in a manner akin to phases in classical liquid crystals, e.g., the nematic and smectic [1]. While the techniques of ultracold atomic physics and quantum optics have enabled explorations of the strongly correlated, many-body physics inherent in, e.g., the Hubbard model [3], lacking has been the ability to create a quantum degenerate Fermi gas with interparticle interactions - such as the strong dipole-dipole interaction (DDI) [4] - capable of inducing analogs to electronic quantum liquid crystals.

Degenerate gases of highly magnetic fermionic atoms, such as ${ }^{161}$ Dy, may shed light on quantum liquid crystal (QLC) physics without unwanted solid state material complexity, disorder, and dynamical lattice distortions. Uniaxial (meta-nematic) [5] and biaxial nematic [6] distortions of the Fermi surface of a harmonically trapped gas in the presence of a polarizing field may be observable as well as meta-nematic and smectic phases in 2D anisotropic optical lattices [7-9]. An exciting prospect lies in the possibility of achieving spontaneous magnetization in dipolar systems coupled with nematic order $[10,11]$. Additionally, the DDI-induced pairing of fermions may lead to "supersolidity" [12] and bond order solids [13].
However, obtaining a quantum degenerate dipolar Fermi gas has been a difficult, unrealized experimental challenge. The highly magnetic fermionic atoms ${ }^{53} \mathrm{Cr}$ (6 Bohr magnetons $\left.\mu_{B}\right)$ and ${ }^{167} \operatorname{Er}\left(7 \mu_{B}\right)$ have yet to be cooled below $10 \mu \mathrm{K}[14,15]$. The fermionic polar molecule ${ }^{40} \mathrm{~K}^{87} \mathrm{Rb}$ $(0.57 \mathrm{D})$ has been cooled to near degeneracy $\left(T / T_{F}=\right.$ 1.4) [16] and loaded into a long-lived lattice while partially polarized (0.2 D) [17], but complexities arising from ultracold chemistry have hampered additional evaporative cooling [16]. In contrast, magnetic fermionic atoms do not undergo chemical reactions and are immune to inelastic dipolar collisions when spin polarized in high magnetic fields $[18,19]$.

The strong, $r^{-3}$ character of the DDI arises in ground state polar molecules through a polarizing electric field that mixes opposite parity states. This electric field breaks rotational symmetry; consequently, observing the full range of true (nonmeta) quantum nematic and fluctuating smectic phases, and their often unusual topological defects, is not possible in systems of fermionic polar molecules, especially in three dimensions. By contrast, highly magnetic atoms exhibit the DDI interaction even in the absence of a polarizing field. Moreover, the magnetic DDI can be tuned from positive to negative [20], which may be important for simulating dense nuclear matter.

Dysprosium's isotopes ${ }^{161}$ Dy and ${ }^{163}$ Dy are the most magnetic fermionic atoms. With a dipole moment of $\mu=$ $10 \mu_{B},{ }^{161}$ Dy provides a DDI length $l_{\mathrm{DDI}}=\mu_{0} \mu^{2} \mathrm{~m} / 4 \pi \hbar^{2}$ that is factors of $\{400,8,2\}$ larger than that of $\left\{{ }^{40} \mathrm{~K},{ }^{53} \mathrm{Cr},{ }^{167} \mathrm{Er}\right\}$. With respect to fully saturated ${ }^{40} \mathrm{~K}^{87} \mathrm{Rb}$ $(0.57 \mathrm{D}),{ }^{161} \mathrm{Dy}$ is 30 times less dipolar for equal densities, but within a factor of 2 if confined in a lattice of less than half the periodicity. Lattices of wavelength $400-500 \mathrm{~nm}$ may be possible with Dy, whereas for molecules, photon 
scattering from rovibronic states at these wavelengths may reduce gas lifetimes. Indeed, ${ }^{161}$ Dy confined in a $450-\mathrm{nm}$ lattice would provide a DDI strength more than 3 times larger than ${ }^{40} \mathrm{~K}^{87} \mathrm{Rb}$ confined in a 1064-nm lattice, if the electric dipole moment is unsaturated $(0.2 \mathrm{D})$ to maintain collisional stability [17]. We estimate that the DDI strength of Dy confined in short-wavelength lattices would be sufficient to observe some of the exotic many-body physics recently proposed $[12,13,21,22]$.

The laser cooling of Dy had long thought to be a challenge due to its complex internal structure and the limited practicability of building repumping lasers: $\sim 140$ metastable states exist between the ground state and the broadest laser cooling transition at $421 \mathrm{~nm}$ [see Fig. 1(a)]. Moreover, an open $f$ shell submerged underneath closed $s$ shells, combined with a large magnetic moment and electrostatic anisotropy from the $L=6$ orbital angular momentum, pose challenges to molecular coupled-channel calculations [23], which could otherwise guide early experiments. The additional hyperfine structure of the fermions, shown in Figs. 1(b) and 1(c), which splits each level into six, complicates matters further.

Despite this vast energy level state space, a repumperless magneto-optical (MOT) technique was able to individually laser cool and trap all five high-abundance isotopes (three bosons, two fermions) to $1 \mathrm{mK}$ using a single laser [24] (see Supplemental Material for details [25]). Moreover, the complex homonuclear molecular potentials-involving 153 Born-Oppenheimer surfaces-and the associated multitude of scattering lengths did not inhibit the efficient
Bose-Einstein condensation (BEC) of spin-polarized ${ }^{164}$ Dy through forced evaporative cooling [18].

However, quantum degeneracy of identical Fermi gases is often more difficult to achieve than BEC because $s$-wave collisions are forbidden due to the requirement that the total wave function for two identical fermions be antisymmetric with respect to particle exchange. Rethermalization from elastic collisions ceases below the threshold for $p$-wave collisions (at typically $10-100 \mu \mathrm{K}$ ), and efficient evaporative cooling can no longer be maintained. Cotrapping mixtures of particles - either as different spin states of the same atom or as mixtures of isotopes or elements-reintroduces $s$-wave collisions, providing a finite elastic cross section for scattering even down to low temperatures. The mixture needs to be stable against inelastic collisions which could add heat or induce trap loss. Evaporating ${ }^{161} \mathrm{Dy}$ in a mixed state of two spins, as proved efficient for ${ }^{40} \mathrm{~K}[26,27]$, would lead to large dipolar relaxation-induced heating even in the presence of small, mG-level fields because the inelastic, single spin-flip cross section $\sigma_{1}=\sigma \zeta\left(k_{f} / k_{i}\right)$ scales strongly with the dipole moment [28]:

$$
\sigma=\frac{8 \pi}{15} F_{1} F_{2}^{2}\left(\frac{\mu_{0} g_{1} g_{2} \mu_{B}^{2} m}{4 \pi \hbar^{2}}\right)^{2} \frac{k_{f}}{k_{i}}
$$

where $F_{1}$ is the spin of the atom whose spin flips $\left(F_{1}=F_{2}\right.$ for identical particles) $g_{i}$ are $g$ factors for atom $i, m$ is mass, and $k_{i}$ and $k_{f}$ are the initial and final momenta. For ${ }^{161}$ Dy $\left({ }^{162}\right.$ Dy $), F=21 / 2(F=J=8)$ and $g_{F}\left(g_{J}\right)=0.95$ (1.24). The function $\zeta\left(k_{f} / k_{i}\right)=\left[1+\epsilon h\left(k_{f} / k_{i}\right)\right]$, where
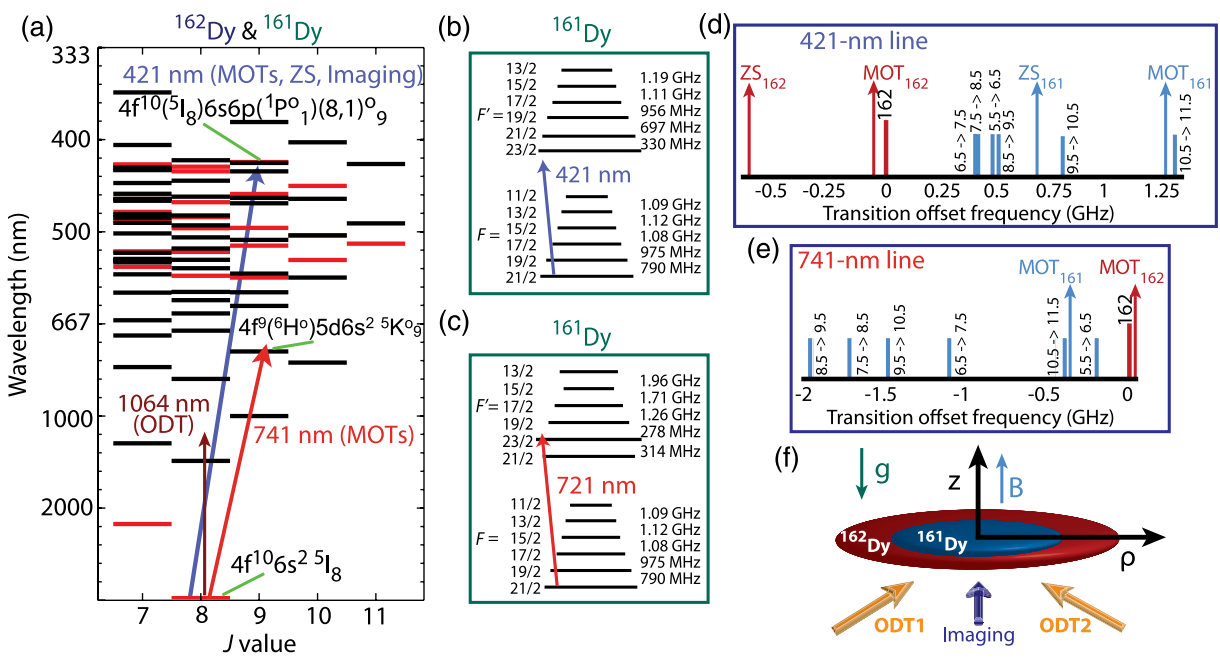

FIG. 1 (color online). (a) Electronic energy level structure for bosonic ${ }^{162}$ Dy (nuclear spin $\left.I=0\right)$ and fermionic ${ }^{161}$ Dy $(I=5 / 2)$, including laser cooling and trapping transitions. (b) Additional ground and excited-state hyperfine structure exists for ${ }^{161} \mathrm{Dy}(F=$ $I+J$, where $J=8$ is the total electronic angular momentum and primes denote the excited states). Shown is the 32-MHz-wide transition at $412 \mathrm{~nm}$ used for the transverse cooling, Zeeman slower, capture MOT, and imaging beams. (c) Blue-detuned, narrowline (1.8 kHz-wide) MOT cooling transition at $741 \mathrm{~nm}$. (d) Spectra of 421-nm ${ }^{162}$ Dy and ${ }^{161}$ Dy (hyperfine) transitions including relative detunings of each MOT and Zeeman slower (ZS) laser. (e) Transition and detuning spectra for MOT on a 741-nm line. (f) Sketch of dual species 1064-nm crossed optical dipole trap (ODT) along with magnetic field $B$ and gravity $g$ orientation. The orthogonal ODT1 and ODT2 beams and the imaging (421-nm) beam are in the $\hat{\rho}$ plane. 
$\epsilon= \pm 1,0$ and $h(x)$ is defined in the Supplemental Material [25], accounts for the contributions of even, odd, or all partial waves to the scattering process.

We choose, therefore, to seek a degenerate dipolar Fermi gas with Dy by sympathetically cooling ${ }^{161}$ Dy with the boson ${ }^{162} \mathrm{Dy}$ while both are spin polarized in their strongmagnetic-field seeking ground states: $\left|F, m_{F}\right\rangle=\mid 21 / 2$, $-21 / 2\rangle$ for ${ }^{161}$ Dy and $\left|J, m_{J}\right\rangle=|8,-8\rangle$ for ${ }^{162} \mathrm{Dy}$. See Figs. 1(a)-1(c) for energy level schemes. We choose to focus this work on isotope ${ }^{161}$ Dy because, unlike the more abundant ${ }^{163} \mathrm{Dy}$, its negative nuclear magnetic moment makes it insusceptible to hyperfine changing collisions after the MOT stage [29]. Preparation of this ultracold Bose-Fermi mixture- the first such mixture for a strongly dipolar species-builds on our single-species technique [18] for Bose condensing ${ }^{164}$ Dy and relies on the laser cooling and trapping of two isotopes before loading both into an ODT for forced evaporative cooling. We sketch here the experimental procedure; further details are provided in the supplement.

Isotopes ${ }^{161}$ Dy and ${ }^{162}$ Dy are collected sequentially in a repumperless MOT operating on the 421-nm transition [24] [Fig. 1(d)], with final MOT populations of $N=2 \times 10^{7}$ and $4 \times 10^{7}$, respectively. Next, simultaneous narrow-line, blue-detuned MOTs [18,30] cool both isotopes to $10 \mu \mathrm{K}$ via the 741-nm transition [Fig. 1(e)] for $5 \mathrm{~s}$ to allow any remaining metastable atoms to decay to the ground state. The blue-detuned MOTs also serve to spin polarize $[18,30]$ both isotopes to their maximally high-field-seeking (metastable) states $m_{F}=+F\left(m_{J}=+J\right)$ for ${ }^{161}$ Dy $\left({ }^{162} \mathrm{Dy}\right)$.

The blue-detuned MOTs of the two isotopes can be spatially separated due to the dependence of the MOTs' positions on laser detuning $[18,30]$. This allows the isotopes to be sequentially loaded into the 1064-nm ODT1 in Fig. 1(f), which is aligned above the ${ }^{161}$ Dy MOT but below the ${ }^{162}$ Dy MOT. First ${ }^{162}$ Dy, and then ${ }^{161}$ Dy, is loaded into ODT1 by shifting the quadrupole center with a vertical bias field. All 741-nm light is extinguished before the spin of both isotopes are rotated via radiofrequency (rf) adiabatic rapid passage (ARP) into their absolute ground states $m_{F}=-F\left(m_{J}=-J\right)$ for ${ }^{161}$ Dy $\left({ }^{162} \mathrm{Dy}\right)$. The ODT1 populations of ${ }^{161}$ Dy and ${ }^{162}$ Dy are both initially $1 \times 10^{6}$ before plain evaporation cools the gases to $1-2 \mu \mathrm{K}$ within 1 s. A $0.9 \mathrm{G}$ field is applied close to the trap axis of symmetry $\hat{z}$ throughout plain and forced evaporation. This provides a $\Delta m=1$ Zeeman shift equivalent to 50 (70) $\mu \mathrm{K}$ for ${ }^{161} \mathrm{Dy}\left({ }^{162} \mathrm{Dy}\right)$. Because this is much larger than the temperatures of the gases, the field serves to maintain spin polarization while stabilizing the strongly dipolar ${ }^{162}$ Dy Bose gas against collapse as its phase-space density increases [18].

Magnetic Stern-Gerlach measurements and observations of fluorescence versus polarization are consistent with a rf ARP sequence that achieves a high degree of spin purity for each isotope. Remnant population in metastable
Zeeman substates quickly decays to the absolute ground state via dipolar relaxation regardless of collision partner at a rate of $\Gamma \propto \sigma_{1} n \bar{v}=1-10 \mathrm{~s}^{-1}$, where $n$ is the atomic density and $\bar{v}$ is the relative velocity during the plain evaporation stage. [Since $g_{F} F=g_{J} J$, collisions between BoseBose, Bose-Fermi, and Fermi-Fermi pairs result in $\Gamma$ 's of similar magnitude as long as $k_{i} \approx k_{f}$ since $h(x \rightarrow 1) \rightarrow 0$. This condition is fulfilled during plain evaporation due to a low ratio of Zeeman-to-kinetic energy. For example, inelastic dipolar ${ }^{161}{ }^{-1}{ }_{-1}{ }^{161}$ Dy collisions $(\epsilon=-1)$ proceed at rate $\Gamma=1-5 \mathrm{~s}^{-1}$ even in the absence of ${ }^{162}$ Dy.] Thus, a (two-body) collisionally stable mixture of identical bosons and identical fermions is prepared within the $1 \mathrm{~s}$ between spin rotation and forced evaporation.

Subsequently, crossing the elliptical ODT1 with the elliptical optical dipole trap ODT2 [see Fig. 1(f)] forms a crossed-beam optical dipole trap with oblate symmetry and frequencies $\left[f_{x}, f_{y}, f_{z}\right]=[500,580,1800] \mathrm{Hz}$. The independent 1064-nm lasers forming ODT1 and ODT2 have initial powers of 18 and $12 \mathrm{~W}$, respectively. An oblate trap formed from elliptical beams is chosen to ensure stability of the ${ }^{162}$ Dy gas as it nears BEC [18]. Ramping down the optical powers lowers the trap depth and evaporates spinpolarized ${ }^{161}$ Dy to quantum degeneracy; with a $19 \mathrm{~s}$ evaporation, the final trap has frequencies $[180,200,720] \mathrm{Hz}$ and $\bar{\omega} / 2 \pi$ is defined as their geometric mean. Figure 2 shows the density profile of ultracold ${ }^{161} \mathrm{Dy}$, which is more consistent with a Thomas-Fermi (TF) distribution arising from

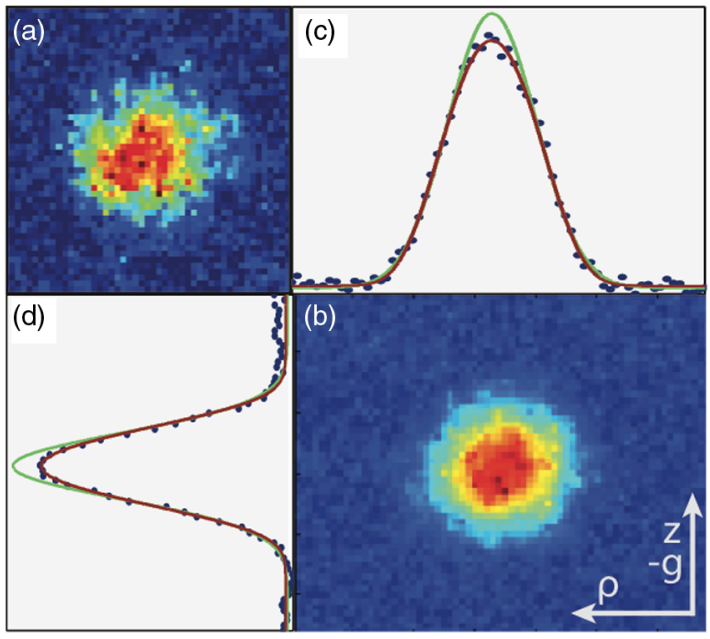

FIG. 2 (color online). (a) Single shot time-of-flight absorption image at $t=6 \mathrm{~ms}$. (b) Average of six images. Density integrations versus $\hat{\rho}$ (c) and $\hat{z}(\mathrm{~d})$. The green curve (larger amplitude) is a Gaussian fit to the data's wings (radius $\sigma=20 \mu \mathrm{m}$ ), while the red curve (smaller amplitude) is a fit to a Thomas-Fermi distribution. Data are consistent with a Thomas-Fermi distribution of $T / T_{F}=0.21(5)$. The Fermi velocity and temperature are $5.6(2) \mathrm{mm} / \mathrm{s}$ and $306(20) \mathrm{nK}$, respectively, and the gas temperature is $64(16) \mathrm{nK}$. The degenerate Fermi gas contains $6.0(6) \times$ $10^{3}$ atoms at peak density $4(1) \times 10^{13} \mathrm{~cm}^{-3}$. 
Fermi-Dirac statistics than a Gaussian arising from a classical, Maxwell-Boltzmann distribution.

Figure 3 shows a collection of such fits as a function of trap population and evaporation time. The $T / T_{F}$ data are extracted from density profiles using three methods: (1) The high momentum wings are fit to a MaxwellBoltzmann distribution to determine $T$ [26,27,31], and the Fermi temperature is calculated from measured trap parameters and population, $k_{B} T_{F}=\hbar \bar{\omega}(6 N)^{1 / 3} ;$ (2) similar to (1), but fitting the entire density profile with a TF distribution to determine $T$; (3) fitting the fugacity to directly extract $T / T_{F}$ (see Supplemental Material [25]). The last method is known to be inaccurate at higher temperatures, while the Gaussian fit tends to overestimate the temperature below $T / T_{F}=1$ [26,27]. The evaporation does not yet seem to reach a plateau in cooling efficiency at $19 \mathrm{~s}$; poor imaging signal to noise hampers measurements at longer evaporation times.

Data in Fig. 3(c) show that thermal equilibrium between the bosons and fermions is maintained throughout the evaporation, and the BEC of ${ }^{162} \mathrm{Dy}$ within the mixture is nearly reached for an evaporation of $19 \mathrm{~s}$. We estimate the corresponding critical temperature $T_{c} \approx 40 \mathrm{nK}$ of cotrapped ${ }^{162}$ Dy by scaling the measured $T_{c} \approx 120 \mathrm{nK}$ of singly trapped ${ }^{162}$ Dy with the cube root of their relative trap populations. ( ${ }^{162}$ Dy has been Bose condensed in the absence of ${ }^{161}$ Dy [32].) The nearly doubly degenerate dipolar Bose-Fermi mixture may lead to interesting dipolar and many-body physics once cooling efficiency improves.

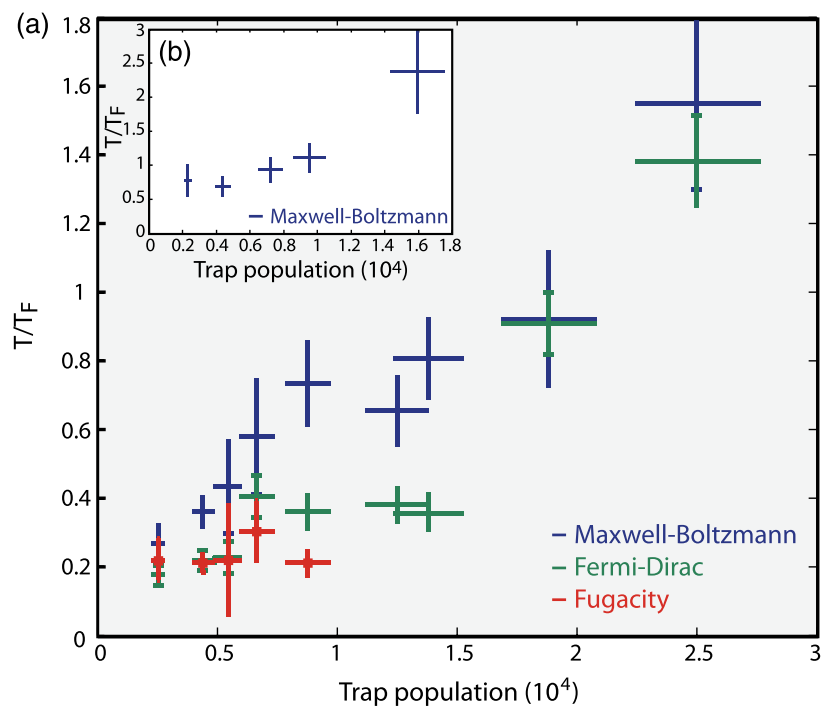

While interacting BECs invert their anisotropic aspect ratio upon time-of-flight expansion, anisotropic degenerate Fermi gases tend to a spherical shape. As the DDI strength increases, the degenerate Fermi gas will expand into a prolate ellipsoid oriented along the magnetization direction regardless of trap aspect ratio. Furthermore, the gas may become unstable when the quantity $\epsilon_{d d}=$ $\mu_{0} \mu^{2}\left(m^{3} \bar{\omega} / 16 \pi^{2} \hbar^{5}\right)^{1 / 2}>1$ [5]. At the lowest attained $T / T_{F}, \epsilon_{d d}=0.2$ for ${ }^{161} \mathrm{Dy}$, and the ratio is $l_{\mathrm{DDI}} / l_{F}$ is 0.05 . This DDI strength should lead to Fermi surface distortions (as yet unmeasured) at the percent level [5]. Both ratios could be enhanced $\sim 3 \times$ by increasing trap frequency using a more spherical confinement-while maintaining the stability of the dipolar Bose gas-and by increasing trap population. While we obtain only several thousand atoms at $T / T_{F}=0.2$, we trap $10^{4}$ atoms at $T / T_{F}=0.5$, comparable, e.g., to the first (unpolarized) ${ }^{173} \mathrm{Yb}$ degenerate Fermi gas [33].

Surprisingly, we achieve the forced evaporative cooling of spin-polarized ${ }^{161}$ Dy without ${ }^{162}$ Dy to $T / T_{F}=0.7$ at $T_{F}=500 \mathrm{nK}$ [see Fig. 3(b)]. As mentioned above, achieving quantum degeneracy with spin-polarized identical fermions alone is usually not possible due to the suppression of elastic scattering below the $p$-wave threshold $50 \mu \mathrm{K}$ [23]. That such a low temperature ratio is achieved may be a novel consequence of the highly dipolar nature of this gas, namely, that a significant elastic cross section persists to low temperatures due to the as yet unobserved phenomenon of universal dipolar scattering [34]. The associated scattering rate is expected to scale as $m^{3 / 2} \mu^{4}$ regardless

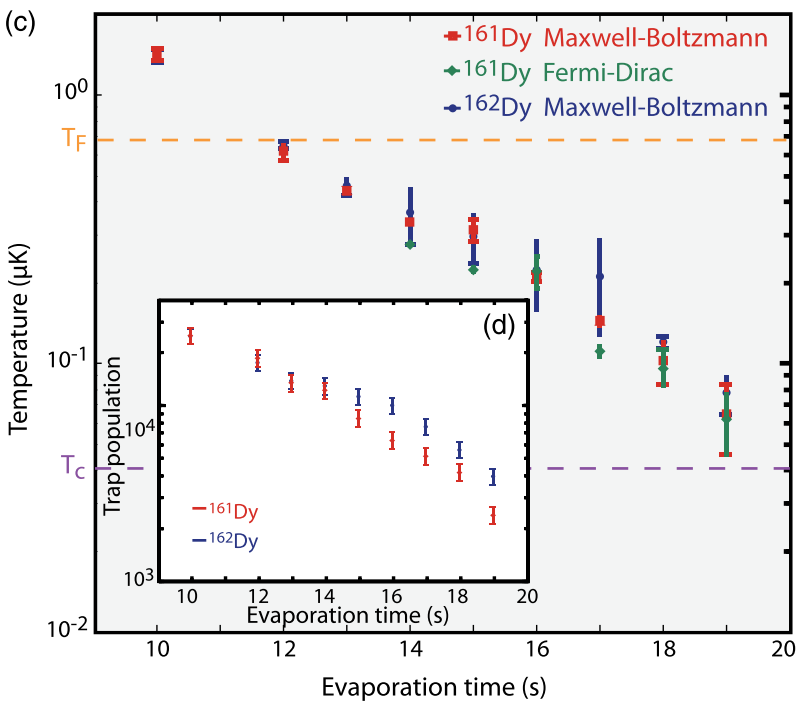

FIG. 3 (color online). (a) Three measures [Maxwell-Boltzmann (dark points), Fermi-Dirac (light-gray points), fugacity (light-grey, square points)] of ${ }^{161}$ Dy $\hat{\rho}$ temperature-to-Fermi temperature $T_{F}$ as trap population decreases due to forced evaporation of the spinpolarized Bose-Fermi mixture. (b) Single isotope evaporative cooling of spin-polarized fermionic ${ }^{161}$ Dy to below $T_{F}$. (c) The dipolar Bose-Fermi mixture remains in thermal contact throughout the evaporation sequence as measured by fits of TOF densities to Gaussian or TF distributions. The orange dashed line demarcates the boundary below which the temperature of ${ }^{161}$ Dy is lower than $T_{F}$. Likewise, the purple dashed line demarcates the temperature below which $T_{c}$ for ${ }^{162}$ Dy Bose degeneracy would be reached given the trap frequencies and population at $19 \mathrm{~s}$. (d) Trap populations of the spin-polarized Bose-Fermi mixture versus evaporation time. 
of the details of the short-range molecular potential. The predicted fermionic Dy universal dipolar cross section, $7.2 \times 10^{-12} \mathrm{~cm}^{2}$, is nearly equal to ${ }^{87} \mathrm{Rb}$ 's $s$-wave cross section and could provide sufficient rethermalization for the evaporative cooling we observe. While future measurements will quantify spin purity, Fermi statistics do not inhibit identical fermions from spin purifying via dipolar relaxation once the rf ARP sequence populates the majority of the atoms in the absolute ground state. We will present these data along with supporting measurements of collisional cross sections and scattering lengths in a future manuscript.

The efficacy of experimental proposals [35] for studying the quantum melting of QLCs - important for better understanding the relationship of incipient electronic stripe order to unconventional superconductivity-may now be investigated using degenerate ${ }^{161} \mathrm{Dy}$ in optical lattices wherein $l_{\mathrm{DDI}} / l_{F}$ can be greatly enhanced [21]. Looking beyond QLC physics, the large spin $F=21 / 2$ of the novel degenerate dipolar Fermi gas presented here opens avenues to explore exotic spinor physics as well as physics associated with strong spin-orbit coupling.

We thank S.-H. Youn for early assistance with experiment construction and J. Bohn, S. Kotochigova, E. Fradkin, N. Goldenfeld, S. Kivelson, G. Baym, C. Wu, H. Zhai, X. Cui, and S. Gopalakrishnan for enlightening discussions. We acknowledge support from the NSF, AFOSR, AROMURI on Quantum Circuits, and the Packard Foundation.

[1] E. Fradkin, S. A. Kivelson, M. J. Lawler, J. P. Eisenstein, and A. P. Mackenzie, Annu. Rev. Condens. Matter Phys. 1, 153 (2010).

[2] E. Fradkin and S. A. Kivelson, Science 327, 155 (2010).

[3] I. Bloch, J. Dalibard, and W. Zwerger, Rev. Mod. Phys. 80, 885 (2008).

[4] T. Lahaye, C. Menotti, L. Santos, M. Lewenstein, and T. Pfau, Rep. Prog. Phys. 72, 126401 (2009).

[5] T. Miyakawa, T. Sogo, and H. Pu, Phys. Rev. A 77, 061603 (2008).

[6] B. M. Fregoso, K. Sun, E. Fradkin, and B. L. Lev, New J. Phys. 11, 103003 (2009).

[7] J. Quintanilla, S. T. Carr, and J. J. Betouras, Phys. Rev. A 79, 031601 (2009).

[8] C. Lin, E. Zhao, and W. V. Liu, Phys. Rev. B 81, 045115 (2010).

[9] K. Sun, C. Wu, and S. Das Sarma, Phys. Rev. B 82, 075105 (2010).

[10] B. M. Fregoso and E. Fradkin, Phys. Rev. Lett. 103, 205301 (2009).

[11] B. M. Fregoso and E. Fradkin, Phys. Rev. B 81, 214443 (2010).

[12] L. He and W. Hofstetter, Phys. Rev. A 83, 053629 (2011).
[13] S. G. Bhongale, L. Mathey, S.-W. Tsai, C. W. Clark, and E. Zhao, Phys. Rev. Lett. 108, 145301 (2012).

[14] R. Chicireanu, A. Pouderous, R. Barbé, B. Laburthe-Tolra, E. Maréchal, L. Vernac, J.-C. Keller, and O. Gorceix, Phys. Rev. A 73, 053406 (2006).

[15] A. J. Berglund, S. A. Lee, and J. J. McClelland, Phys. Rev. A 76, 053418 (2007).

[16] K. K. Ni, S. Ospelkaus, D. Wang, G. Quemener, B. Neyenhuis, M.H.G. de Miranda, J.L. Bohn, J. Ye, and D. S. Jin, Nature (London) 464, 1324 (2010).

[17] A. Chotia, B. Neyenhuis, S. A. Moses, B. Yan, J. P. Covey, M. Foss-Feig, A. M. Rey, D. S. Jin, and J. Ye, Phys. Rev. Lett. 108, 080405 (2012).

[18] M. Lu, N. Q. Burdick, S.-H. Youn, and B.L. Lev, Phys. Rev. Lett. 107, 190401 (2011).

[19] B. Pasquiou, G. Bismut, Q. Beaufils, A. Crubellier, E. Maréchal, P. Pedri, L. Vernac, O. Gorceix, and B. Laburthe-Tolra, Phys. Rev. A 81, 042716 (2010).

[20] S. Giovanazzi, A. Görlitz, and T. Pfau, Phys. Rev. Lett. 89, 130401 (2002).

[21] M. Dalmonte, P. Zoller, and G. Pupillo, Phys. Rev. Lett. 107, 163202 (2011).

[22] A. V. Gorshkov, S. R. Manmana, G. Chen, J. Ye, E. Demler, M. D. Lukin, and A.M. Rey, Phys. Rev. Lett. 107, 115301 (2011).

[23] S. Kotochigova and A. Petrov, Phys. Chem. Chem. Phys. 13, 19165 (2011).

[24] M. Lu, S.-H. Youn, and B. L. Lev, Phys. Rev. Lett. 104, 063001 (2010).

[25] See Supplemental Material at http://link.aps.org/ supplemental/10.1103/PhysRevLett.108.215301 for further descriptions of the laser cooling, trapping and evaporative cooling sequences, as well as information on the fitting routines and evaporation efficiency.

[26] B. DeMarco and D.S. Jin, Science 285, 1703 (1999).

[27] B. DeMarco, Ph.D. thesis, University of Colorado at Boulder, 2001

[28] S. Hensler, J. Werner, A. Griesmaier, P. O. Schmidt, A. Görlitz, T. Pfau, S. Giovanazzi, and K. Rzazewski, Appl. Phys. B 77, 765 (2003).

[29] S.-H. Youn, M. Lu, U. Ray, and B. L. Lev, Phys. Rev. A 82, 043425 (2010).

[30] A. J. Berglund, J. L. Hanssen, and J. J. McClelland, Phys. Rev. Lett. 100, 113002 (2008).

[31] B. J. DeSalvo, M. Yan, P. G. Mickelson, Y. N. Martinez de Escobar, and T. C. Killian, Phys. Rev. Lett. 105, 030402 (2010).

[32] N.Q. Burdick, M. Lu, and B.L. Lev (manuscript in preparation).

[33] T. Fukuhara, Y. Takasu, M. Kumakura, and Y. Takahashi, Phys. Rev. Lett. 98, 030401 (2007).

[34] J. L. Bohn, M. Cavagnero, and C. Ticknor, New J. Phys. 11, 055039 (2009).

[35] S. A. Kivelson, I. P. Bindloss, E. Fradkin, V. Oganesyan, J. M. Tranquada, A. Kapitulnik, and C. Howald, Rev. Mod. Phys. 75, 1201 (2003). 